\title{
Immune responses against oxidized LDL as possible targets for prevention of atherosclerosis in systemic lupus erythematosus
}

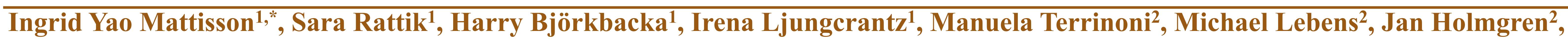
Gunilla Nordin Fredrikson', Birgitta Gullstrand ${ }^{3}$, Anders A Bengtsson ${ }^{3}$, Jan Nilsson', Maria Wigren ${ }^{1}$

${ }^{1}$ Department of Clinical Sciences Malmö, Skåne University Hospital, Lund University, Malmö, Sweden, ${ }^{2}$ Department of Microbiology and Immunology, Gothenburg University, Gothenburg, Sweden, ${ }^{3}$ Department of Clinical Sciences Lund, Lund University, Lund, Sweden

\section{AIM}

To investigate if targeting the oxidized LDL (oxLDL) autoimmunity can affect atherosclerosis in systemic lupus erythematosus (SLE) to reduce the cardiovascular disease (CVD) prevalence.

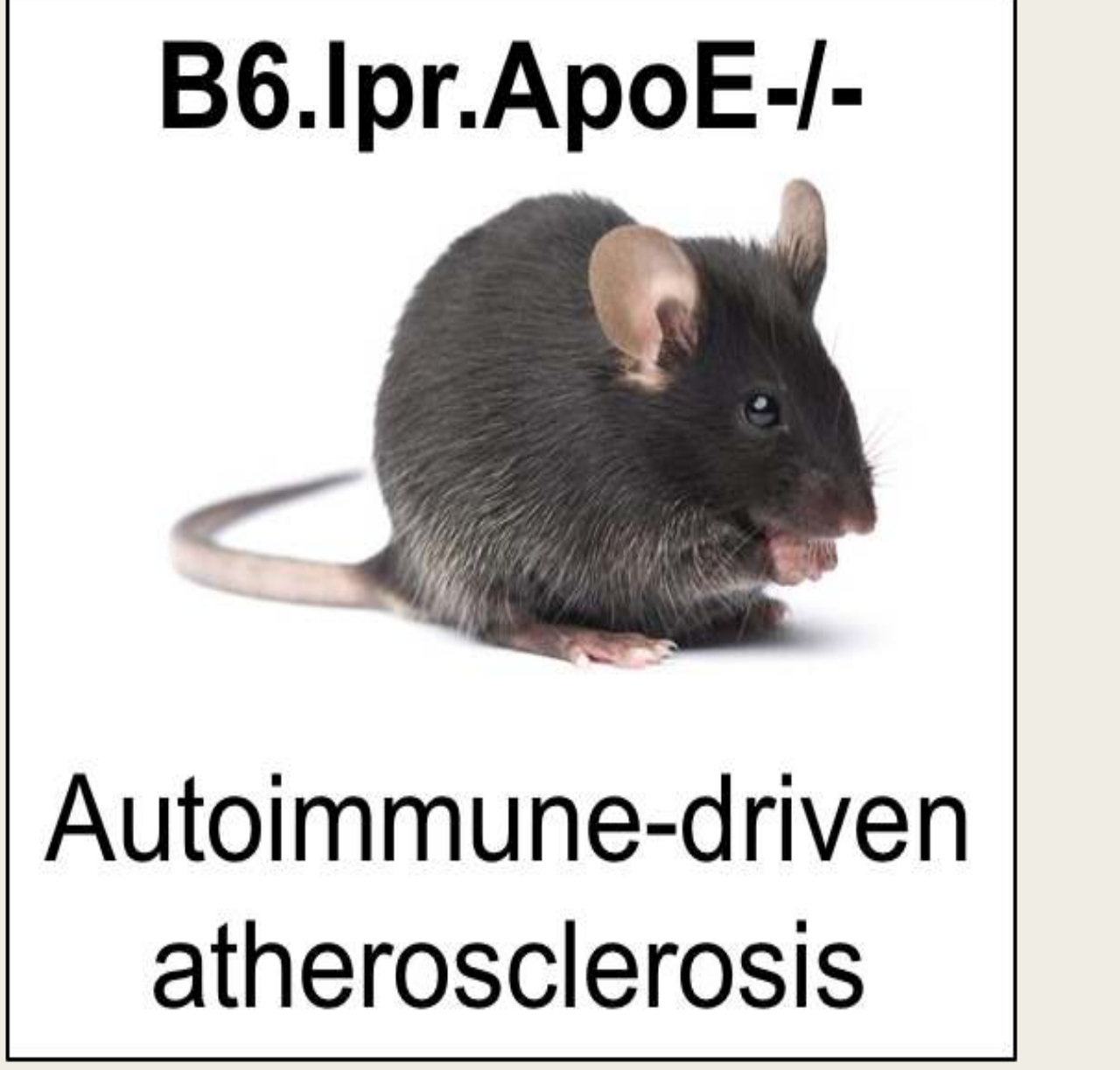

\section{Hypothesis}

OxLDL can be modulated with immunotherapeutical strategies in a hypercholesterolemic lupus-like mouse model model (B6.lpr.ApoE $\mathrm{E}^{-/}$).

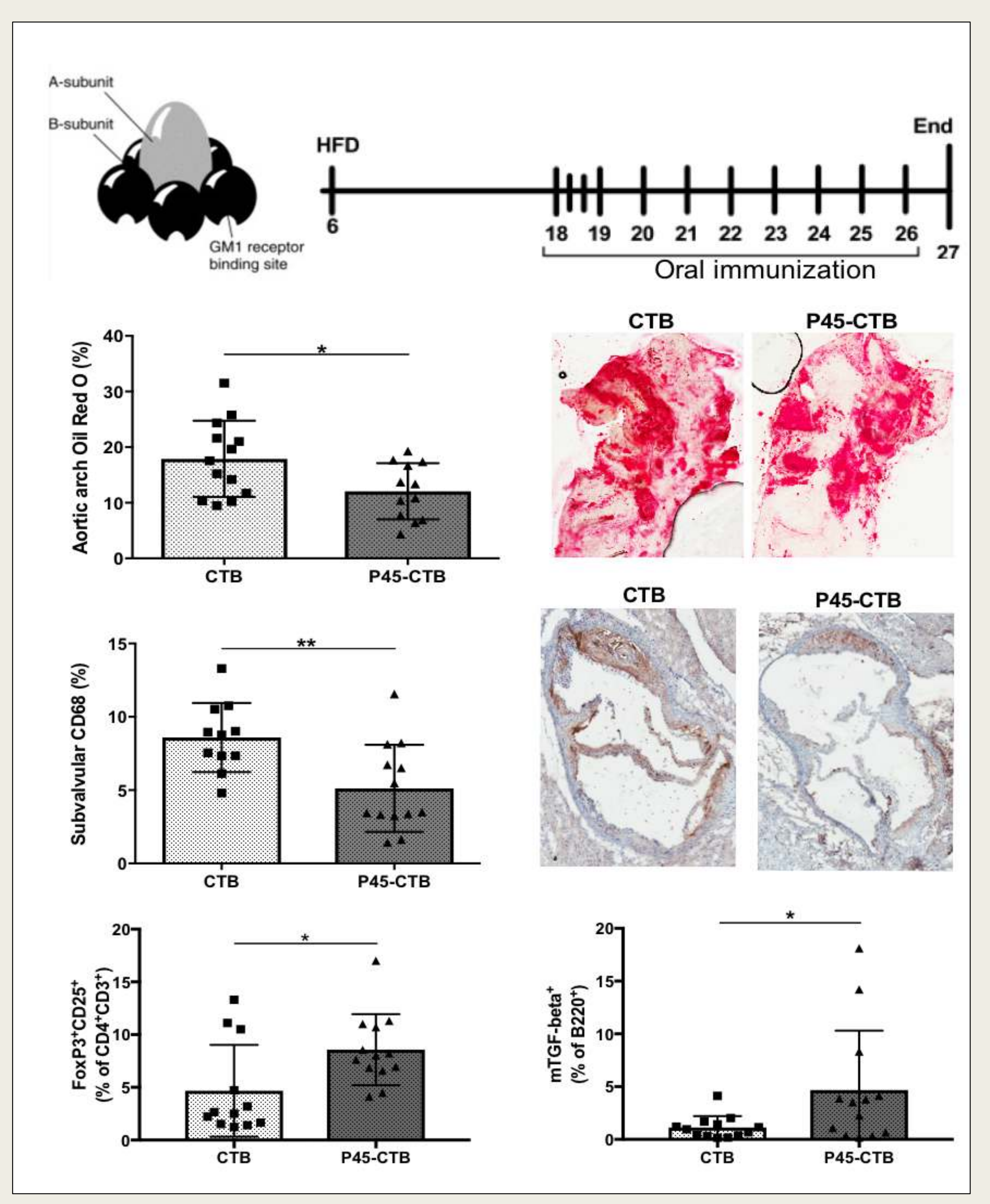

\section{Background}

It is clinically relevant to develop novel therapies to modulate the autoimmune responses driving atherosclerosis. Patients suffering from SLE are at increased risk of developing CVD and traditional therapies including statins provide insufficient protection. Impaired removal of apoptotic material is a common pathogenic mechanism in both SLE and

atherosclerosis and is considered to be a key factor in the development of autoimmunity.

\section{Methods}

We assess two immunomodulatory approaches to target the oxLDL autoantigen using the B6.lpr.ApoE ${ }^{-/-}$mouse model, similar to the premature atherosclerosis observed in SLE patients.


\section{Results}

Mucosal tolerance induction with a fusion protein between apolipoprotein B-100 peptide p45 (amino acids 661-680) and cholera toxin B-subunit (P45-CTB) increased regulatory $\mathrm{T}$ cells and $\mathrm{B}$ cells in mesenteric lymph nodes and reduced plaque development in the aorta by $33 \%$ compared to cholera toxin B-subunit (CTB) alone. Antibody-treatment with the human recombinant MDA-p45 specific IgG, binding to the
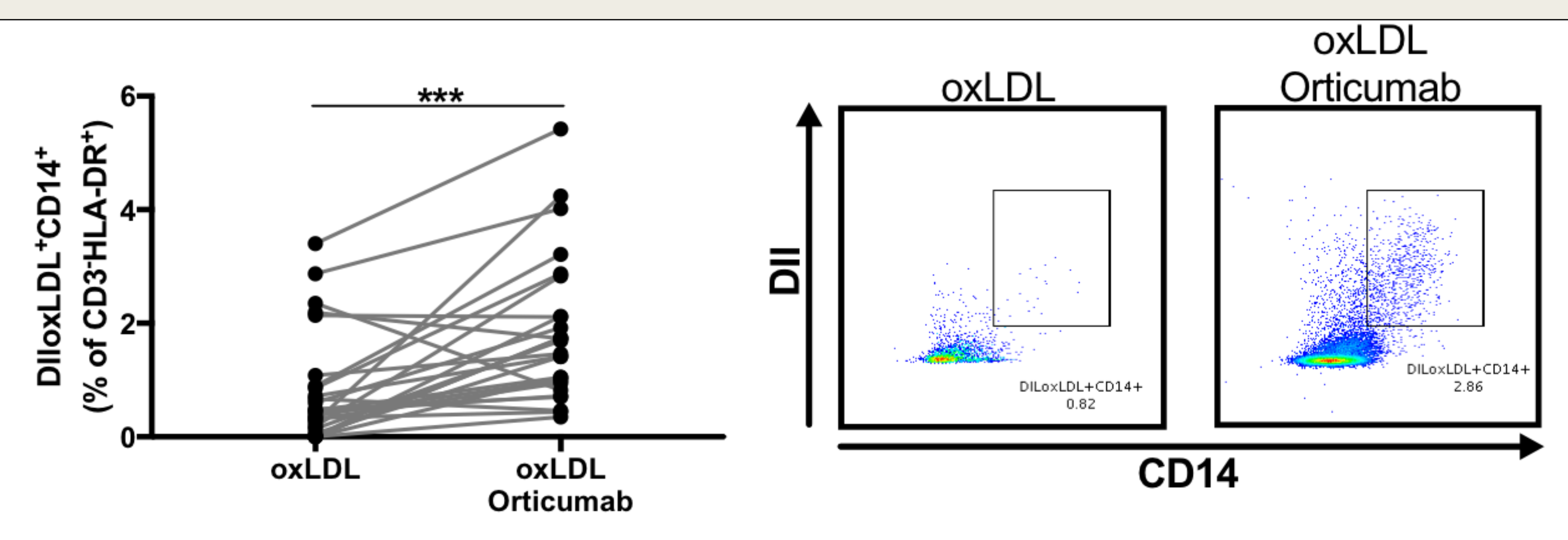

inhibitory Fcrreceptor IIB, reduced aortic atherosclerosis by $43 \%$, subvalvular plaque area by $50 \%$ and the macrophage content by $31 \%$. Additionally, this antibody facitilated active removal of circulating oxLDL successfully through $\mathrm{CD} 14^{+}$cells from SLE PBMC in vitro, suggesting an induction in systemic clearance of the autoantigen.

\section{Conclusion}

The present study provides experimental and clinical support for oxLDL as a possible target for prevention of cardiovascular complications in SLE. 